\title{
THE INCORPORATION OF WORK HARDENING AND REDUNDANT WORK IN ROD-DRAWING ANALYSES
}

\author{
A. G. Atriss and R. M. Caddell \\ Department of Mechanical Engineering, The University of Michigan, \\ Ann Arbor, Michigan, 48104, U.S.A.
}

(Received 20 June 1967)

Summary-With few exceptions, motal deformation analyses employ a constant yield stress (rigid, perfectly plastic metal) which ignores strain hardening, or a "mean" yield stress which attempts to accommodate strain hardening in a simplified manner. Since strain hardening is of interest here, little reference will be made to a rigid, plastic type of behavior.

The first part of this paper dernonstrates that the use of a mean yield stress underestimates the working loads (or stresses) needed to draw metal through conical dies as compared to those loads predicted by more "exact" analyses. In this context "exact" refers to those solutions obtained by incorporating the strain hardening relationship in the governing "force balance" differential equations prior to the integration of the said equations. It is shown, however, that the error introduced by the use of a mean yield stress is no more than some 8 per cent for conditions that typify actual practice. Since analyses of other metal-working processes, such as rolling and extrusion, employ the same sort of differential equation, it is felt that these results are applicable there also.

The second part of this paper shows that redundant work in rod drawing may be approached either from considerations of the mechanical properties that result after the metal is drawn or from considerations of the stress necessary to draw down the rod. Contrary to what is impliod in the literature, it is shown that these two approaches lead to different interpretations of the "redundant work factor". Relationships aro given between the two for metals that are assumed to strain harden in certain simple ways.

\section{NOTATION}

a semi-angle of die cone

b) diameter of rod before drawing

$d$ diameter of rod after drawing

$r$ decimal reduction of area $=1-\left(\frac{d}{D}\right)^{2}$

$\mu$ gross, average coefficient of friction between rod and die

$\sigma_{d}$ drawing stress with no back tension

$Y_{n}$ mean uniaxial yield stress (dependent upon strain hardening and redundant work)

B $\mu \cot \alpha$

$\varepsilon_{n} \quad$ homogeneous true strain $=2 \ln \left(\frac{D}{d}\right)=\ln \left(\frac{1}{1-r}\right)$

$\varepsilon_{0} \quad$ true strain induced prior to drawing

$a, b$ constants associated with linear strain hardening (i.e. $Y=a+b \varepsilon$ )

$\sigma_{0}, m$ constants associated with power-law strain hardening (i.e. $Y=\sigma_{0} \varepsilon^{m}$ )

$\sigma$ true stress

$f, g, h$ constants associated with exponential strain hardening [i.e. $Y=f-g \exp (-h \varepsilon)]$ 


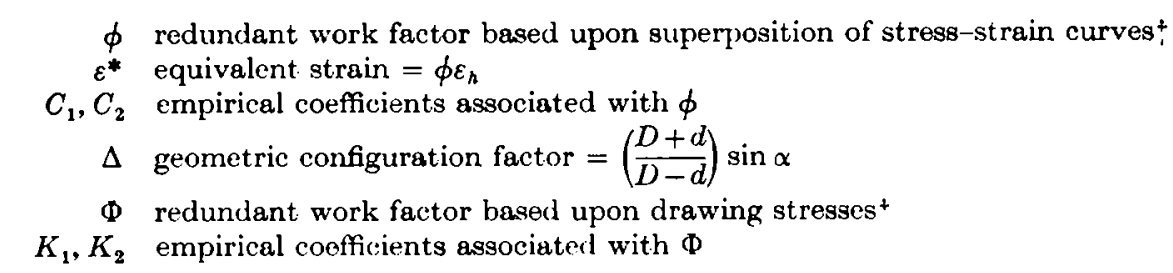

\section{Introduction}

\section{PART I. DRAWING ANALYSES}

MucH of the previous work devoted to drawing metal through conical dies has been summarized by MacLellan ${ }^{1}$ and, later, Wistreich ${ }^{2}$; together these papers contain an extensive list of references relating to this area of study. Previous publications which have some bearing on this present paper will be referred to, otherwise the reader can $\operatorname{check}^{1,2}$ for a more complete source of information.

Although Shield ${ }^{3}$ has provided a solution to axisymmetric drawing through conical dies of semi-infinite length, Wistreich ${ }^{2}$ points out that the results have limited practical value because of restrictions regarding end effects. In fact, no rigorous and truly acceptable solution to the plasticity problem posed by axisymmetric drawing has yet been found. The literature does contain a number of analyses, however, that provide a definite insight to this problem; some employ the use of two-dimensional plane-strain drawing as a guideline but these results do not, of course, provide a one-to-one correspondence with the axisymmetric case. The work of Hill and Tupper ${ }^{1}$, Whitton ${ }^{5}$ and Green ${ }^{6}$ demonstrate (though not exclusively) some of the important previous studies. For the development of the objectives of this present paper, the theory originally presented by Sachs ${ }^{7}$ will be of principal concern. Modifications of this theory (e.g. Körber and Eichinger ${ }^{8}$ ) will be ignored except for reference to the work of Davis and Dokos ${ }^{9}$. Additionally, use will be made of the theory developed by Hill and Tupper ${ }^{4}$ (see Baron and Thompson ${ }^{10}$ ) which was based upon a slip-line field analysis. It should be noted that this same result had earlier been proposed by Sachs and Van I Iorn ${ }^{11}$, who used a force balance analysis.

\section{General considerations}

Throughout this paper it is assumed that there is no back tension applied to the metal as it is drawn through a conical die. In terms of the parameters of semi-angle of the die $(\alpha)$, reduction of area $(r)$ and an average value of coefficient of friction at the die-workpiece interfaco $(\mu)$, expressions for the drawing stress $\left(\sigma_{d}\right)$ have been developed as follows:

$$
\begin{gathered}
\sigma_{d}=Y_{m}(1+B) \ln \left(\frac{1}{1-r}\right)=Y_{m}(1+B) \varepsilon_{h} \\
\sigma_{\lrcorner}=Y_{m}\left(\frac{1+B}{B}\right)\left[1-(1-r)^{B}\right]=Y_{m}\left(\frac{1+B}{B}\right)\left[1-\exp \left(-B \varepsilon_{h}\right)\right]
\end{gathered}
$$

where $r$ is the decimal equivalent to the percent reduction of area, $\varepsilon_{n}$ is the homogeneous, logarithmic strain besed upon the reduction of area, $Y_{m}$ is some constant yield stress, and

† One referee has suggested the adoption of the following torminolngy:

$\phi$-redundant deformation factor (based upon superposition of stress-strain curves);

$\Phi-$ redundant work factor (based upon drawing stresses).

The authors feel that this suggestion has definite merit as it might lead to far greater clarity in future discussions of "redundant work factors". 
$B=\mu \cot \alpha$. If one notes that

$$
1-\exp (-B \varepsilon)=B \varepsilon-\frac{(B \varepsilon)^{2}}{2 !}+\ldots
$$

it becomes apparent that as $B \varepsilon$ becomes small, equation (2) eventually reduces to equation (1).

An even more pertinent point to note is that equation (2) was derived from a stress theory based upon the following differential equation, which can allow for variation of the flow stress with strain:

$$
\frac{\mathrm{d} \sigma_{d}}{\mathrm{~d} \varepsilon}+B \sigma_{d}=Y(\varepsilon)(1+B)
$$

If $Y(\varepsilon)$ is taken as some mean (or constant) flow stress, the solution of (4) leads to (2). If, however, the functional dependence of $Y$ on $\varepsilon$ is known, that is, if strain hardening can be handled rationally, the use of a mean yield stress would be unnecessary. Equation (4) could bo solved [using the integrating factor $\exp (B \varepsilon)$ ] to give

$$
\sigma_{d} \exp \left(B \varepsilon_{h}\right)=(1+B) \int_{0}^{\delta_{n}} Y(\varepsilon) \exp (B \varepsilon) \mathrm{d} \varepsilon
$$

For simplicity, it is assumed throughout this paper (unless specifically stated otherwise) that the material before drawing contains no prior work hardening, hence, the lower limit of zero in equation (5). Inserting the function $Y(\varepsilon)$ inside the integral sign would then enable one to obtain 8 solution that accounts for strain hardening. The obvious problem, of course, is to choose a proper function for $Y(\varepsilon)$. Davis and Dokos ${ }^{\circ}$ have provided one such solution.

Pertinent assumptions made in the stress formulation that lead to equation (2) are well documented elsewhere ${ }^{10}$ and need not be repeated here.

Hill and Tuppert used a slip-line anelysis for plane-strain drawing as a model for the axisymmetric case. They proposed a method for handling strain hardening that, in essence, provides a correction to equation (1). A very thorough coverage of plane-strain drawing, and suggested extensions of past theories, has been published by Green.

\section{The effect of strain hardening $\dagger$}

In the approach of Hill and Tuppert, where no differential equation exists after the formulation of the problem, the effect of strain hardening can be handled only by employing a type of mean yield stress for the range of equivalent strain induced by the drawing operation. With the Sachs treatment, this restriction is not confronted; in fact, Davis and Dokos provided a solution by assuming the metal was a rigid, linear, work-hardening solid (i.e. $Y=a+b \varepsilon$, where $a$ and $b$ are constants). Using this relationship in equation (5) they found the following:

$$
\sigma_{d}=\left(\frac{1+B}{B}\right)\left\{\left[1-\exp \left(-B \varepsilon_{h}\right)\right]\left(a-\frac{b}{B}\right)+b \varepsilon_{h}\right\}
$$

The present authors feel that the oft-quoted but less often used power law form of strain hardening very well typifies the strain hardening behavior of many common metals. Here, $Y=\sigma_{0} \varepsilon^{m}$, where $\sigma_{0}$ and $m$ are considered as constent properties of the metal. +

$\dagger$ The influence of strain-rate and temperature are not considered in this paper.

+ In the form $Y=\sigma_{0} \varepsilon^{m}$ it is implicit that this equation predicts the behavior of a metal containing no initial cold work. If the metal had been subjected to prior work hardening equal to a strain $\varepsilon_{0}$, then its subsequent behavior is depicted by $Y=\sigma_{0}\left(\varepsilon_{0}+\varepsilon\right)^{m}$. In this manner, $\sigma_{0}$ and $m$ are considered to be material constants which do not change with the condition of initial strain hardening.

If, howover, for an initially strained motal, a typical tensile test is conducted and $\log \sigma$ is plotted against $\log \varepsilon$ (i.e. $\varepsilon_{0}$ is ignored), the points always lie on a shallow conceve-up curve with no straight portion; this is due to the nonlinear $\log$ scales. To quote average values of $\sigma_{0}$ and $m$ for various states of strain hardening, from plots which are definitely not straight lines, seems very questionable practice. 
Voce ${ }^{12,13}$ proposed the stress-strain relationship, $Y=f-g \exp (-h \varepsilon)$, where $f, g$ and $h$ are arbitrary constants. Although this provides a flexible arrangement that permits close fitting to many curves, the simpler powor law form describes the strain hardening behavior of metals sufficiently well. To illustrate the principal point under consideration in this part of the paper, it is unnecessary to employ a variety of possible forms depicting strainhardening characteristics. 'Therefore, further discussion will be restricted solely to the two forms that follow

$$
\begin{array}{ll}
Y=a+b \varepsilon & \text { (line:tr work hardening) } \\
Y=\sigma_{0} \varepsilon^{m} & \text { (power-law work hardening) }
\end{array}
$$

If equation (8) were substituted in (5), one would get

$$
\sigma_{d}=(1+B)\left[\exp \left(-B \varepsilon_{h}\right)\right] \int_{0}^{e_{n}} \sigma_{0} \varepsilon^{m} \exp (B \varepsilon) \mathrm{d} \varepsilon
$$

Since $m$ is always fractional $(0<m<0 \cdot 6$ for materials whose experimental values of $m$ are quoted in the literature), equation (9) cannot be solved in closed form and so is best exprossed as

$$
\sigma_{d}=\sigma_{0}(1+B)\left[\exp \left(-B \varepsilon_{n}\right)\right] \sum_{n=0}^{\infty} \frac{B^{n} \varepsilon^{m+n+1}}{n !(m+n+1)}
$$

Fortunately, practical values of $(B \varepsilon)$ are usually less than unity and rapid convergence of the series occurs after expanding about four terms.

When an analytical form for the stress-strain curve is not known, an analog computer can be usefully employed to integrato equation (4), as recently shown by Rowe ${ }^{14}$. Chapter 12 of his book gives examples of the technique.

\section{The mean yield stress method}

Much of the previous work related to rod or wire drawing entails the use of a mean yield stress of the drawn metal in order to solve for the drawing stress. Such a procedure, in essence, attempts to consider the effects of strain hardening by correcting for it after the fact. One might certainly ask just how accurate is this approach compared to one that considers strain hardening in the basic governing equation. (Note that in order to present this clearly, one must revert to an equation such as (4) which is based on a stress analysis.)

For the linear work-hardening material, the mean yield stress from equation (7) would be

$$
Y_{m}=\frac{1}{\varepsilon_{n}} \int_{0}^{e_{n}}(a+b \varepsilon) \mathrm{d} \varepsilon=a+\frac{b \varepsilon_{n}}{2}
$$

and inserting (11) in (5) would give

$$
\sigma_{d}=\left(\frac{1+B}{B}\right)\left(a+\frac{b \varepsilon_{h}}{2}\right)\left[1-\exp \left(-B \varepsilon_{h}\right)\right]
$$

For the power-law form of strain hardening, the mean yield stress is found from

$$
Y_{m}=\frac{1}{\varepsilon_{h}} \int_{0}^{e_{\Lambda}} \sigma_{0} \varepsilon^{m} \mathrm{~d} \varepsilon=\frac{\sigma_{0} \varepsilon_{h}^{m}}{m+1}
$$

that is, the mean yield stress is simply the flow stress at a strain $\varepsilon_{\lambda}$, divided by the factor $(m+1)$. When the results of (13) are combined with (5) one gets

$$
\sigma_{d}=\left(\frac{1+B}{B}\right)\left(\frac{\sigma_{0} \varepsilon_{n}^{m}}{m+1}\right)\left[1-\exp \left(-B \varepsilon_{h}\right)\right]
$$

Comparison of (12) with (6) and (14) with (10) shows that the use of a mean yield stress does not produce a solution equivalent to the more exact analysis.

Rather than computing differences at this point, let us rearrange (6) as follows:

$$
\sigma_{d}=\left(\frac{1+B}{B}\right)\left[1-\exp \left(-B \varepsilon_{h}\right)\right]\left(a+b \varepsilon_{h}\left(\frac{1}{1-\exp \left(-B \varepsilon_{n}\right)}-\frac{1}{B \varepsilon_{h}}\right\}\right)
$$


Making use of the approximation (see Green ${ }^{6}$ )

$$
\frac{1}{1-\exp (-B \varepsilon)} \approx \frac{1}{2}\left(1+\frac{2}{B \varepsilon}+\frac{B \varepsilon}{6}\right)
$$

which is accurate to 1 per cent for $B \varepsilon=2$ and $\frac{1}{10}$ per cent for $B \varepsilon=1$, equation (15) maybe reduced to

$$
\sigma_{d}=\left(\frac{1+B}{B}\right)\left[1-\exp \left(-B \varepsilon_{\mathrm{h}}\right)\right]\left[a+b \varepsilon_{\lambda}\left(\frac{1}{2}+\frac{B \varepsilon_{\lambda}}{12}\right)\right]
$$

As $B \varepsilon$ becomes smaller, equation (16) derived from the exact analysis reduces to the "mean" yield stress expression as given by equation (12).

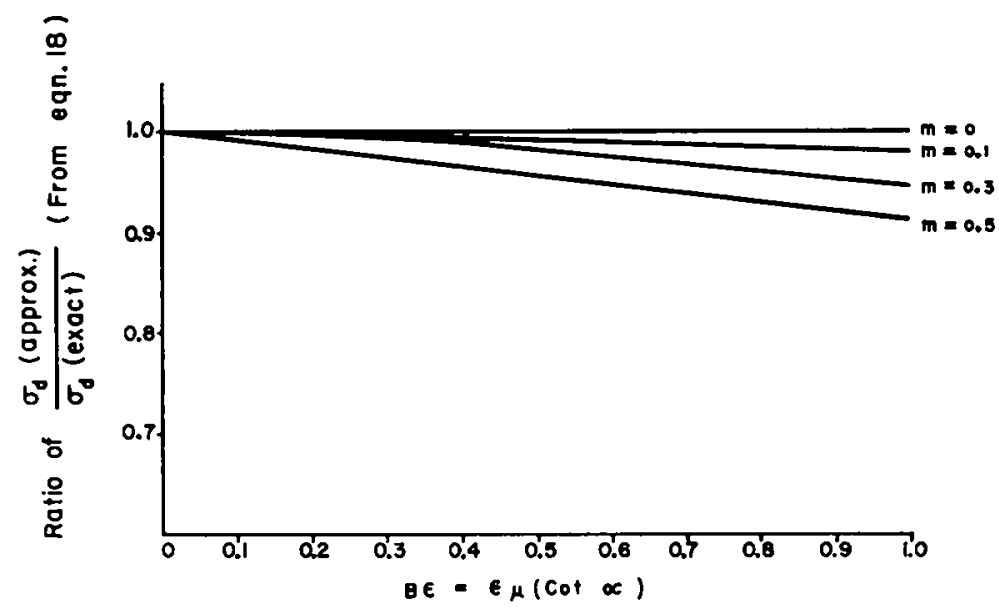

F19. 1. Comparison of predicted drawing stress using a "mean" yield stress and a more "exact" analysis for various combinations of $(B \varepsilon)$ and strain-hardening rates.

This same general finding can be shown by comparing (14) with (10) although the mathematical manipulation becomes lengthy and cumbersome. It is more convenient to work with the ratio of the "exact" to "approximate" drawing stress [i.e. ratio of equation (10) to (14)].

Simplifying gives

$$
\frac{\sigma_{d} \text { from }(10)}{\sigma_{d} \text { from }(14)}=\frac{(1+B)\left[\exp -\left(B \varepsilon_{h}\right)\right] \sigma_{0} \sum_{n=0}^{\infty}\left[\frac{B^{n} \varepsilon_{h}^{(m+n+1)}}{n !(m+n+1)}\right]}{\left(\frac{1+B}{B}\right) \frac{\sigma_{0} \varepsilon_{h}^{m}}{m+1}\left[1-\exp \left(-B \varepsilon_{n}\right)\right]}
$$

$$
\frac{\sigma_{d} \text { from (10) }}{\sigma_{d} \text { from (14) }}=\frac{(m+1) \sum_{n=0}^{\infty}\left[\frac{\left(B \varepsilon_{n}\right)^{n+1}}{n !(m+n+1)}\right]}{\exp \left(B \varepsilon_{n}\right)-1}
$$

If one takes the reciprocal form of (18), in order to judge how closely the ratio of approximate stress to exact stress approaches unity, the results as a function of $(B \varepsilon)$ are shown in Fig. 1. Several different work-hardening exponents have been chosen and it may be observed that the greatest deviation occurs at large values of $(B \varepsilon)$ with large values of $m$. It was found sufficient to use a maximum of five terms of the series in (18) since rapid convergence occurs; under the least favourable combination of conditions $(B \varepsilon=1$ and $m=0 \cdot 5$ ), the use of a mean yield stress provides predicted drawing stresses within 10 per cent as compared to the more exact solution.

From the above one can infer that regardless of the shape of the strese-atrain curve, the use of a mean yield stress will always underestimate the magnitude of the required drawing stress. In this light, the results presented in Fig. 12.5 of the text by Rowe ${ }^{14}$ are 
of interest and lend confirmation to these conclusions. There, comparison is made between a computer solution of equation (5) and a manual solution of equation (14); with reasonable practical values of $B \varepsilon$ (up to $\approx 0.5$ ) excellent agreement was found. Fig. 1 of this paper would predict a drawing stress from equation (14) to be about 98 per cent of that predicted from equation (10), since Rowe's stress-strain data for mild steel (Fig. 12.2a of his text) fit the equation $\sigma=107,000 \varepsilon^{0.23}$.

Emphasis must be placed on the observation that Fig. 1 was derived for a material containing no initial strain hardening: if this condition is not met, it is obvious that the lower limit on the various integrals is finite and not zero. Although no calculations are given here, it may, however. be shown that the differences between predictions based upon "mean" yield stress versus "exact" analysis diminishes with increasing initial cold work. This comes about bocause the stress-strain curves, depicting strain hardening characteristics, tend to flatten because of the influence of $\varepsilon_{0}$, and the behavior approaches that of a rigid, plastic material (that is, loosely speaking, $m$ approaches zero).

In closing this section of the paper, it seems proper to note one important observation. Due to the perhaps fortuitous fact that practical magnitudes of $m$ and $(B \varepsilon)$ fall within the range of those shown in Fig. 1, the use of a mean yield stress will generally provide sensible predictions of drawing stress. (This, of course, implies that values of $\mu$, as used in these calculations, are realistic.) Thus, the extra mathematical manipulation that enters with more exact analyses is, in general, unjustified. Up to this point, reference to "redundant work" has been avoided; this will now be discussed.

\section{PART II. REDUNDANT WORK IN ROD DRAWING}

\section{General considerations}

When a rod of diameter $D$ is reduced to a diameter $d$ by drawing through a die, it suffers a strain which is actually greater than would be expected merely from considerations of homogeneous plastic deformation. The origin of this effect may be found in the "redundant work" performed in the die, where the external constraint causes appreciable internal distortion of the workpiece beyond that strictly necessary for shape change alone. Although, as Hundy and Singer ${ }^{15}$ have shown, the induced strain can be quite inhomogeneous under certain conditions, being greater on the outside of the workpiece than at the center, it is nevertheless convenient to write the strain actually imposed as $\varepsilon^{*}=\phi \varepsilon_{h}$, where $\phi$ is called the "redundant work factor".

The existence of redundant work will, of course, necessarily increase drawing stresses, above the sort of values that are predicted by the equations given in the preceding sections. This provides one approach towards defining the redundant work factor and will be discussed subsequently. This second part of the paper really concerns itself with the compatibility of two different ways of defining the redundant work factor.

\section{Redundant work from mechanical properties}

Because of the "enhanced strain" in the drawn rod, it has, for example, a greater yield strength than homogeneous deformation would predict, and this suggests one way of determining $\phi$ exporimentally for different combinations of materials, reductions and die angles. If the stress-strain curve of the drawn metal be superposed on the stress-strain curve of the undrawn metal, $\phi$ may be evaluated directly from the ratio of "equivalent strain" $\left(\varepsilon^{*}\right)$ to the homogeneous strain $\left(\varepsilon_{n}\right)$, where $\varepsilon^{*}$ is the abscissa value by which the curve of the drawn material must be shifted to line up with the curve of the undrawn material. Although such curve-fitting can be quite capricious, the technique has been used quite often.

A rather nice point presents itself in the measurement of the mechanical properties of the drawn rod because the question arises whether one should make standard tensile test pieces with cut-away gauge sections, which removes the more highly worked outer layers, or whether one should test the rod "whole". Certainly, standard tensile pieces give lower values for the yield strength of the drawn metal when redundant work has been large. Consequently, it seems better to tensile test the whole drawn rod, hoping that necking does not occur in the grips. If it does, one has to test anew, although a simple way to 
minimize fracture in the grips is to make steps on the rod before drawing; this gives a test piece with more highly worked ends. (See Caddell and Atkins ${ }^{16}$.)

From yield stress messurements, two main parameters seem to influence the magnitude of $\phi$, namely, the angle of the die, and the percent reduction taken. It has been shown that the relationship between these quantities may be expressed as follows:

$$
\phi=C_{1}+C_{2} \Delta
$$

where $\Delta=\left(\frac{D+d}{D-d}\right) \sin \alpha$. This may be interpreted physically as the ratio of the mean cross-section diameter of the conical die to the frustum slant length of contact between the die and workpiece. Both $C_{1}$ and $C_{2}$ vary with the work-hardening characteristics of the metal being drawn; although $C_{1}$ does not vary appreciably from a value of about 0.9 , $C_{2}$ can vary considerably. In a recent study of redundant work using commercially pure aluminum, Armco iron, an austenitic stainless steel, and an age-hardening aluminum alloy, ${ }^{16}$ none of which contained initial cold work, empirical relationships for these coefficients were found as follows:

$$
\begin{aligned}
& C_{1}=3.70 m^{0.28} \sigma^{-0.10} \\
& C_{2}=0.48 m^{0.78} \sigma^{-0.054}
\end{aligned}
$$

where $\sigma_{0}$ and $m$ have been defined previously.

Other relations have been reported for $\phi$ (e.g. Linicus and Sach ${ }^{17} \dagger$ ), but such expressions may be shown to be equivalent to equation (19) as indicated in another work. ${ }^{10}$ Moreover, the $\Delta$ form as in (19) appears to be preferable because of the analogy with the more exact form for two-dimensional strip drawing. (See, for example, Green ${ }^{\circ}$.)

As to the friction at the die-work interface, if it does influence $\phi$, it may well be a second-order effect. Wistreich ${ }^{18}$ showed that the yield strengths of wires, drawn down by the same amounte, were similar whether drawing was done dry or well lubricated (of course, the drawing stresees and die pressures were quite different). However, later in that paper, and in his review paper, ${ }^{2}$ Wistreich indicates that redundant work should be influenced by friction. Different opinions on this point may be found in the literature, but let it suffice to observe that there is to date no complete agreement. In the study ${ }^{16}$ that led to equations (20) and (21), the possible effects of friction were not investigated since only one condition of lubrication was employed.

\section{Redundant work from drawing stresses}

Much of the published work on wire-drawing has concerned itself more with predicting the stress necessary to draw rod through dies, rather than considering the subsequent mechanical properties of the drawn wire. The empiricel theories of Korber and Eichinger ${ }^{8}$ and Siebel ${ }^{19}$, which are discussed by Wistreich ${ }^{2}$, are the only analyses which formally take redundant deformation into account. The expressions for drewing stress are somewhat like equation (2) with an additional term for redundant work added on. However, both Wistreich ${ }^{2}$ and Whitton ${ }^{5}$ point out the potential shortcomings of both of these ompirical theories.

In Part I of this paper, some typical analyses relating drawing stress with die angle, percent reduction taken and die-workpiece frictional effects were discussed. Those analyses would necessarily give underestimates for drawing stresses because the effort in

$\dagger$ Linicus and Sachs, by measuring mechanical properties of drawn wire, give

$$
\phi \approx 1+\frac{\Delta}{8}-\frac{5 \alpha}{8}
$$

for initially annealed brass. Again, Wistreich2, by measuring drawing stresses, gives

$$
\Phi=0.87+\frac{\Delta}{4}-\frac{\alpha}{2}
$$

for initially worked copper. It may be noted that this latter expression is developed from a drawing-stress equation [equation (27) of Wistreich ${ }^{18}$ ] using the approximation $\Delta \approx 4 \alpha / \varepsilon$. 
performing redundant work had not been taken into account. (This discrepancy, of course, has nothing to do with the fact that the theories may give different predictions for particular combinations of $\mu$ and $\varepsilon$, and also slightly different answers depending on the way work hardening is treated.)

Despite these last problems, to make up for the fact that $\sigma_{d}$ would be underestimated by one's inability to cope with redundant work in a force-balance analysis, the druwing. stress equations are often multiplied by a factor we shall call $\Phi$. For reasons that will bo clear later, this parameter is distinguished from $\phi$, although tho literature does not do this.

Consequently, for a rigid-plastic, non-work-hardening solid, the "real" drawing stress is said to be either equation (1) or (2) multiplied by some empirical $D$. When this is extended to a work-hardening material, Hill's treatment employs a mean yield stress. again applied to equation (1), but with the Sachs approach one has a choice as to whether equations (6) or (12) or equations (10) or (14) might be used (of course, other variations are possible depending upon the form of the strain-hardening behavior leemed most appropriate).

If one measures $\mu$ (e.g. MacLellan's ${ }^{20}$ split-die technique as employed by Wistreich ${ }^{1 s}$ and Yang ${ }^{21}$ ), then $\Phi$ may be deduced by comparison of mexured and predicted values of drawing stress. In this way, $\Phi$ can be related to $\alpha$ and $r$, much like the expression for $\phi$ in equation (19), i.e.

$$
\left.\Phi=K_{1}+K_{2}\right\lrcorner
$$

It is at once apparent, however, that the magnitude of $\Phi$ may depend markedly on the particular analysis used to predict the drawing stress. Perhaps of even greater concern is that without measured values of friction, one really has two unknowns, $\Phi$ and $\mu$. For this reason, there has been the tendency to employ the various drawing-stress equations to solve for $\mu$ under those conditions where $\Phi$ is approximately unity (i.e. combinations of large reductions and small die angles).

\section{Compatibility of $\phi$ and $\Phi$}

The literature would seem to imply that $\phi$ and $\Phi$ are the same parameter, but in a recent st udy, ${ }^{16}$ where various ways of est imating redundant work were investigated, it became apparent that these were not identical. This camo about as follows. Consider that $\phi$ is our basic definition in terms of the actual strain induced by drawing being greater than the homogeneous strain. Further, assume that $\phi$ is known for various combinations of die angle and reduction. Then, instead of using a factor like $\Phi$ to molify the drawing. stress equations to compensate for redundant work, let us attempt to incorporate the effects of redundant work in the derivation of the drawing-stress equation.

The yield stress of a metal which has been subjected to the homogeneous strain, $\varepsilon_{h}$, will actually be the flow stress at $\phi \varepsilon_{h}$ because of redundant work. Employing the definition of mean yield stress used previously, this means

$$
Y_{m}=\frac{1}{\phi \varepsilon_{h}} \int_{0}^{\phi \varepsilon_{h}} Y(\varepsilon) \mathrm{d} \varepsilon \text { rather than } Y_{m}=\frac{1}{\varepsilon_{h}} \int_{0}^{\varepsilon_{h}} Y(\varepsilon) \mathrm{d} \varepsilon
$$

Returning to the form of Sachs's equation (i) and considering the effect of redundant work on the yield stress, there results

$$
\sigma_{d} \exp \left(B \phi \varepsilon_{h}\right)=(1+B) \int_{0}^{\phi_{\varepsilon_{h}}} Y(\varepsilon) \exp (I B \varepsilon) \mathrm{d} \varepsilon
$$

Now in Part I of this paper it was shown that even under unfavorable conditions (large reductions with metals that strain harden severely), the error introduced by using a mean yield stress was usually woll under 10 per cent as compared to a more exact approach. It is felt that these same comments are appropriate here, so instead of using expressions for $\boldsymbol{Y}(\varepsilon)$, such as equations (7) or (8), in equation (24) the more simplified approach using a mean yield stress will be followed. Certainly, the comparison of $\phi$ with $\Phi$ does not appear to be invalidated by using this simpler concept, since it is felt that differences in drawingstress predictions do not affect the $\Phi-\phi$ relationship. 
For the strain-hardening behavior given by (11) and (13), and considering equivalent strain rather than homogeneous strain, we may write

$$
\begin{gathered}
Y_{m}=a+\frac{b \phi \varepsilon_{h}}{2} \\
Y_{n}=\frac{\sigma_{0} \phi^{m} \varepsilon_{h}^{m}}{m+1}
\end{gathered}
$$

These may be substituted directly in equation (2) to give

and

$$
\sigma_{d}=\left(\frac{1+B}{B}\right)\left(a+\frac{b \phi \varepsilon_{h}}{2}\right)\left[1-\exp \left(-B \phi \varepsilon_{h}\right)\right]
$$

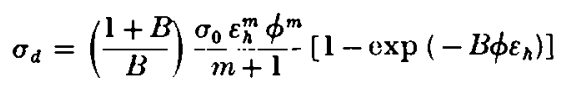

If, however, one used the factor $\Phi$ to correct for redundant work, the corresponding expressions [i.e. equations (12) and (14)] would be

and

$$
\sigma_{t}=\Phi\left(\frac{1+B}{B}\right)\left(a+\frac{b \varepsilon_{h}}{2}\right)\left[1-\exp \left(-B \varepsilon_{h}\right)\right]
$$

$$
\sigma_{d}=\Phi\left(\frac{1+B}{B}\right) \frac{\sigma_{0}}{m+1} \frac{\varepsilon_{h}^{m}}{m+1}\left[1-\exp \left(-B \varepsilon_{h}\right)\right]
$$

Now if $\phi$ and $\Phi$ are identical then equivalence must exist between (27) and (29) and between (28) and (30). To demonstrate that this is not the case, it will be sufficient to consider only (28) and (30).

Combining these expressions, one gets

$$
\Phi=\left[\frac{1-\exp \left(-\phi B \varepsilon_{h}\right)}{1-\exp \left(-B \varepsilon_{h}\right)}\right] \phi^{m}
$$

As $(B \varepsilon)$ becomes small, as in equation (3), equation (31) approaches

$$
\Phi=\phi^{m+1}
$$

Clearly, $\Phi=\phi$ only when $m=0$ (rigid, plastic material); in fact, when $(B \varepsilon)$ is not small, $\Phi \neq \phi$ even if $m=0$. The same conclusion (i.o. $\Phi \neq \phi$ ) will result if a comparison is made between equations (27) and (29); the details are left to the reader.

To provide a quantitative comparison, the rolationship in equation (31) could be used, but it seems more appropriate to introduce the parameter $\Delta$ since expressions like (19) and (22) are generally found in the literature. In order to pursue this, $(B E)$ in (31) must be replaced by an equivalent expression containing $\Delta$. This can be approximated as follows:

$$
(B \varepsilon) \approx \mu \cot \alpha\left(\frac{4 \sin \alpha}{\Delta}\right)=\frac{4 \mu}{\Delta} \approx \frac{\cos \alpha}{\Delta}
$$

It will be shown subsequently that the above approximation introduces no serious error even up to values of $\alpha$ that are much larger than those used in practice. Introducing (33) into (31) gives

$$
\Phi=\left[\frac{1-\exp \left(\frac{-4 \mu \phi}{\Delta}\right)}{1-\exp \left(\frac{-4}{\Delta} \underline{\mu}\right)}\right] \phi^{m}
$$

In using equation (34), values of $\Delta$ from 2 to 20 were first substituted into equations patterned after (19). The empirical expressions used for 303 stainless steel and commercially pure aluminum were roported in another study ${ }^{16}$ the pertinent values for $m(0.52$ for the stainless and 0.23 for the aluminum) were also included in that same study. 
These expressions for $\phi$ were as follows:

$$
\begin{array}{ll}
\phi=0.87+0.15 \Delta & \text { (stainless) } \\
\phi=0.89+0.092 \Delta & \text { (aluminum) }
\end{array}
$$

'To check the influence of $\mu$ on the $\Phi-\phi$ comparison, values of $0,0.05,0 \cdot 10,0.20$ and $0 \cdot 50$ were employed. This range would seem to encompass practically all values of $\mu$ for drawing that are reported in the literature. The complete sot of values determined for the range of $\Delta$ and $\mu$ mentioned above are listed in Tablo 1. 'To justify the approximation

\begin{tabular}{|c|c|c|c|c|c|c|c|c|}
\hline \multirow[b]{2}{*}{$\Delta$} & \multirow[b]{2}{*}{$\phi$} & \multicolumn{7}{|c|}{$\Phi$} \\
\hline & & $\mu \rightarrow 0$ & 0.05 & $0 \cdot 10$ & $0 \cdot 10 \dagger$ & $0 \cdot 20$ & 0.50 & $0.50 \dagger$ \\
\hline \multicolumn{9}{|c|}{303 Stainless steel } \\
\hline 2 & $1 \cdot 17$ & 1.27 & $1 \cdot 27$ & $1 \cdot 26$ & $1 \cdot 24$ & $1 \cdot 24$ & $1 \cdot 19$ & $1 \cdot 19$ \\
\hline 3 & 1.32 & 1.52 & 1.50 & $1 \cdot \tilde{1} 1$ & 1.50 & 1.47 & $1 \cdot 36$ & $1 \cdot 40$ \\
\hline 5 & $1 \cdot 62$ & 2.09 & 2.08 & 2.06 & 2.03 & $2 \cdot 01$ & $1 \cdot 90$ & 1.88 \\
\hline 7 & $1 \cdot 92$ & $2 \cdot 69$ & $2 \cdot 71$ & $2 \cdot 71$ & $2 \cdot 64$ & $2 \cdot 60$ & $2 \cdot 39$ & $2 \cdot 43$ \\
\hline 10 & $2 \cdot 37$ & $3 \cdot 70$ & $3 \cdot 68$ & $3 \cdot 70$ & $3 \cdot 62$ & 3.54 & $3 \cdot 24$ & $3 \cdot 28$ \\
\hline 16 & $3 \cdot 27$ & $6 \cdot 0$ & $6 \cdot 17$ & $5 \cdot 8$ & $5 \cdot 62$ & $5 \cdot 73$ & $5 \cdot 30$ & $5 \cdot 4 \mathrm{~s}$ \\
\hline 20 & $3 \cdot 87$ & $7 \cdot 80$ & $7 \cdot 67$ & $7 \cdot 60$ & $7 \cdot 52$ & $7 \cdot 65$ & $6 \cdot 91$ & $6 \cdot 90$ \\
\hline \multicolumn{9}{|c|}{ Commercially pure aluminum } \\
\hline 2 & $1 \cdot 07$ & 1.09 & $1 \cdot 08$ & $1 \cdot 10$ & 1.08 & 1.08 & $1 \cdot 06$ & $1 \cdot 05$ \\
\hline 3 & $1 \cdot 17$ & $1 \cdot 21$ & $1 \cdot 20$ & $1 \cdot 21$ & $1 \cdot 20$ & $1 \cdot 19$ & $1 \cdot 14$ & $1 \cdot 16$ \\
\hline 5 & $1 \cdot 35$ & 1.45 & $1 \cdot 48$ & $1 \cdot 44$ & $1 \cdot 41$ & $1 \cdot 41$ & $1 \cdot 36$ & 1.37 \\
\hline 7 & 1.53 & $1 \cdot 69$ & $1 \cdot 66$ & 1.67 & $1 \cdot 66$ & 1.65 & 1.58 & $1 \cdot 58$ \\
\hline 10 & 1.81 & 2.08 & 2.07 & $2 \cdot 11$ & 2.07 & $2 \cdot 04$ & 1.91 & 1.93 \\
\hline 16 & $2 \cdot 36$ & $2 \cdot 87$ & $2 \cdot 90$ & $2 \cdot 7 x$ & $2 \cdot 65$ & $2 \cdot 80$ & $2 \cdot 61$ & 2.70 \\
\hline 20 & $2 \cdot 73$ & $3 \cdot 45$ & $3 \cdot 38$ & $3 \cdot 40$ & 3.43 & $3 \cdot 42$ & $3 \cdot 20$ & 3.20 \\
\hline
\end{tabular}

TABle 1. Redundant work factors for various values of $\Delta$ and $\mu$

$\dagger \Phi$ calculated from equation (31) where $I B \varepsilon=4 \mu \cos \alpha / \Delta$. All other calculations for $\Phi$ based upon equation (34).

indicated in (33), additional calculations were made. By choosing a large value of $\alpha\left(22 \cdot 5^{\circ}\right)$, using $4 \mu \cos \alpha / \Delta$ rather than $4 \mu / \Delta$, and employing two of the values of $\mu$ used previously $(0.10$ and 0.50$)$, the pertinent values of $\Phi$ were found for the same range of $\Delta$ values. These findings are also included in Table 1, and since all calculations were performed to slide-rule accuracy, the approximato form given by (34) certainly appears to introduce no significant error. It should be noted that for $\mu=0, \Phi=\phi^{m+1}$ regurdless of the magnitude of $\alpha$.

The plots shown in Figs. 2 and 3 include the extreme lines which bound $\Phi$ versus $\Delta$ for all of the values listed in 'Table 1. 'T'o plot every point would, possibly, lead to a loss of clarity. The $\phi$ versus $\Delta$ plots on these figures are simply the graphical form of equations (35) and (36).

Now strictly speaking, the $\Phi-\Delta$ plots, calculated as a function of $\phi$, are not straight lines and the departure from linearity is more severe with the stainless steel (this has a larger strain-hardening exponent, $m$ ). However, if we take the liberty of assuming that these plots are reasonably linear up to $\Delta$ values of $10 \mathrm{or}$ so, we can write the following expressions :

$$
\begin{array}{ll}
\Phi \approx 0.87+0.27 \Delta & \text { (stainless) } \\
\Phi \approx 0.89+0.12 \Delta & \text { (aluminum) }
\end{array}
$$

From Figs. 2 and 3, or a comparison of (37) and (38) with (35) and (36), it is obvious that $\Phi$ and $\phi$ are not the same parameter. As pointed out by Green ${ }^{6}$, Wistreich's work ${ }^{18}$ 
indicated that $\Phi$ is overestimated when annealed metals are subjected to low reductions (i.e. large $\Delta$ values). The rather drastic increase of $\Phi$ with increasing $\Delta$, as shown in Figs. 2 and 3 , may be interpreted as support of that observation.

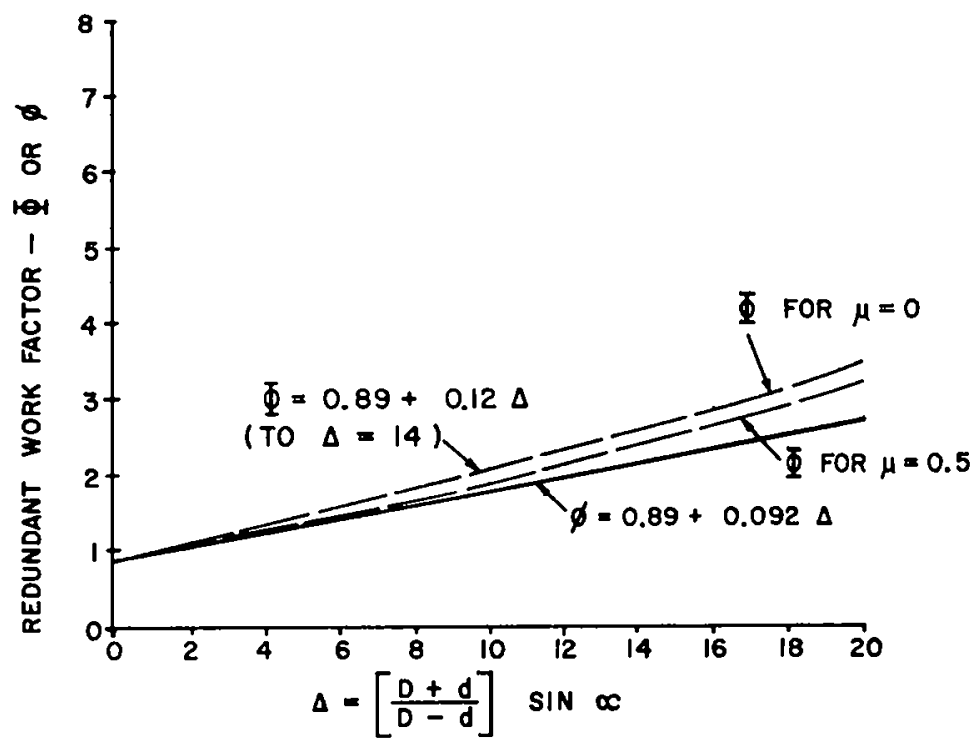

Fia. 2. Comparison of the redundant work factors, $\Phi$ and $\phi$, for commercially pure aluminum.

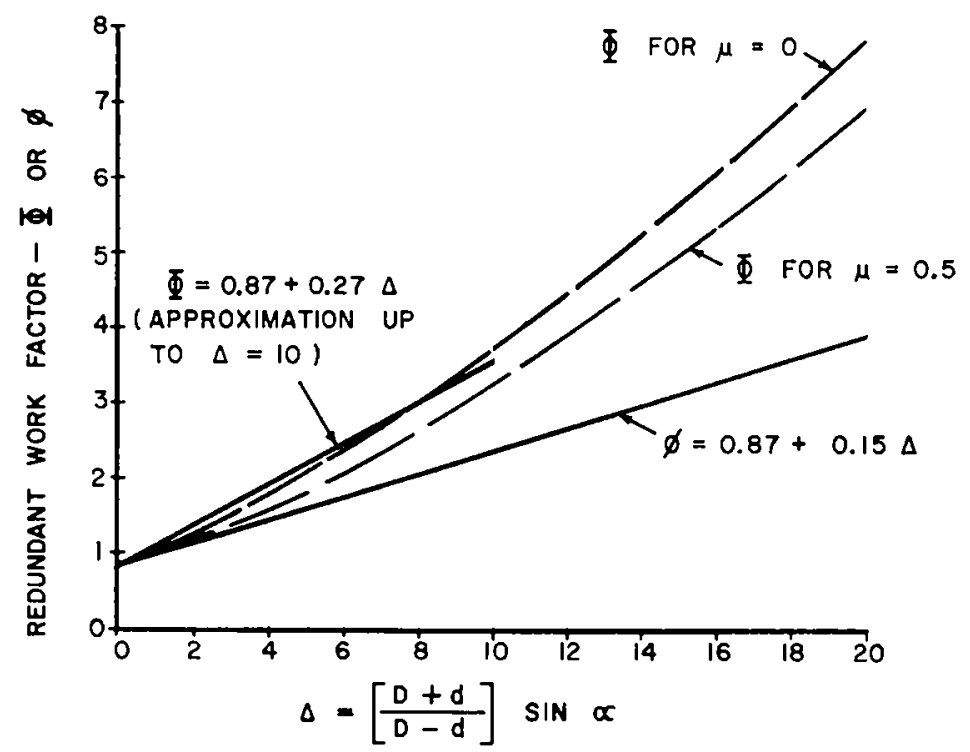

Fic. 3. Comparison of the redundant work factors, $\Phi$ and $\phi$, for type 303 stainless steel.

At present, there seems to be no obvious way to produce an explicit relationship between $C_{9}$ and some representative value for $K_{2}$, although for these two metals the constants $C_{1}$ and $K_{1}$ are practically identical. Further experimental work appears necessary before this concept can be extended. 
One observation is included for consideration. 'The apparent insensitivity of $\Phi$ with regard to $\mu$ as soen in Table 1 (certainly up to values of $\mu=0 \cdot 20$ ) tempts one to suggest that friction has only a small influence on redundant work, although it must be notod that these results depend upon one analysis used for determining the drawing stress.

\section{DISCUSSION}

The full presentation under fiection 4 (earlier was based upon materials containing no strain hardening prior to drawing. In the study ${ }^{16}$ that produced equations (20), (21), (35) and (36), no work was done with metals that were strained before being drawn, so the influence of $\varepsilon_{0}$ [from $\sigma=\sigma_{0}\left(\varepsilon_{0}+\varepsilon\right)^{m}$ ] on $C_{1}$ and $C_{2}$ in equation (19) is not known. From a qualitative viewpoint, however, the effect. of prior cold work is to reduce $m$ and increase $\sigma_{0} . \dagger$ If this follows, then from equations (20) and (21) the tendency would be for $C_{2}$ to diminish more rapidly than would $C_{1}$. In essence, this implies that the effects of redundant work are less pronounced on prior worked metals than on non-worked metals; it would follow, therefore, that the plots of $\Phi$ and $\phi$ versus $\Delta$ would become flatter and the difference between $\Phi$ and $\phi$ would lessen, the more the prior cold work.

'To pursue this point, let us refer to the comprehensive set of data reported by Rowe't and Johnson ${ }^{23}$. Their work entailed the use of copper, brass, mild steel and aluminum, all of which had been initially strain hardened 25 per cent prior to drawing. Apparently, they did not employ originally annealed materials in that study. For all of these metals, they found a best-fit relationship as follows $\ddagger$

$$
\Phi=0.88+(0 \cdot 19-0 \cdot 22) \Delta
$$

Since there is at present no experimental data that would permit one to correct equations (35) and (36) to account for any possible effect of initial strain hardening, it is not fully possible to make a complete comparison between $\Phi-\Delta$ relationships predicted from equations (31), (35) and (36) and Rowe and Johnson's experimental $\Phi-\perp$ form per equation (39). However, a rough comparison will now be made, but this at tempt must not be construed to be anything more than culalitative in nature.

Table 2. Comparisox of $\phi$ aNi $\Phi$ for folr anNealed MEtals

\begin{tabular}{|c|c|c|c|c|c|c|}
\hline Material & $\sigma_{0}^{\dagger}$ & $m^{+}$ & $c_{1}+$ & $c_{2+}^{+}$ & $\phi \S$ & $\Phi$ \\
\hline Copper & 72,000 & 0.50 & 1.00 & $0 \cdot 16$ & $1 \cdot 95$ & $2 \cdot 62$ \\
\hline $70-30$ Brass & 105,000 & 0.52 & 0.98 & $0 \cdot 16$ & 1.94 & $2 \cdot 62$ \\
\hline Mild steel & 100,000 & $0 \cdot 23$ & 0.78 & 0.09 & $1 \cdot 30$ & $1 \cdot 34$ \\
\hline Aluminum & 26,000 & 0.23 & 0.88 & 0.09 & $1 \cdot 31$ & $1 \cdot 34$ \\
\hline
\end{tabular}

+ Values selected from l)atsko $)^{25}$.

† Calculated from equations (20) and (21).

$\$$ From equation (19), using $\Delta$ of 6 and $c_{1}$ and $c_{2}$ as tabulated above.

$\|$ From equation (34), using $\Delta$ of $6, \mu$ of 0.10 and $\phi$ as tabulated above.

In Tuble 2, typical values for $\sigma_{0}$ and $m$ are shown for the four metals used by Johnson ${ }^{23}$; these values pertain to the annealed metals subjected to standard tensile tests. Assuming the reliability of equations (20) and (21) is reasonable, the corresponding values of $C_{1}$ and $C_{2}$ may be readily calculated; these are listed in this same table. Since the maximum value of $\Delta$ used by these other authors ${ }^{14}, 23$ would correspond to a value of 6 in our definition, this value was used to determine the magnitude of $\phi$ as per equation (19) with $C_{1}$ and $C_{2}$ defined in Table 2. Because the results in Table 1 illustrated the apparent

$\dagger$ See footnote on page 17 and comments on the top of page 21.

$\ddagger$ Rowe ${ }^{14}$ employs a parameter, $\Delta$, that is one-quarter the $\Delta$ value used in this paper. The present authors have converted accordingly as Rowe ${ }^{24}$ gave a coefficient of $0.76-0.88$ in equation (39). 
insensitivity of $\Phi$ with regard to $\mu, \mathbf{a}$ value of $\mu=0 \cdot 1$ was chosen and $\Phi$ was then determined for each of the four annealed materials using equation (34) (at a value of $\Delta=6$ also). By assuming that the $\Phi-\Delta$ relationship is reasonably linear up to $\Delta=6$ (see Figs. 2 and 3 ), then the slope of the line can be determined between $\Delta=0$ and $\Delta=6$. It turns out that two distinct lines are found; one fits the copper and brass while the other fits the steel and aluminum. These were as follows

$$
\begin{aligned}
& \text { Copper and brass: } \Phi \approx 1 \cdot 0+0.27 \Delta \\
& \text { Steel and aluminum: } \Phi \approx 0 \cdot 8+0.09 \Delta
\end{aligned}
$$

The reason why one would expect such differences can be seen in the significant influence of $m$ and the rather modest effect of $\sigma_{0}$ on the coefficients $C_{1}$ and $C_{2}$. Although beyond this point it is not presently possible to convert the annealed relations of (40) and (41) to similar forms for the prior-worked metals, one would qualitatively expect the slopes to become shallower [i.o. lower coefficients of the $\Delta$ term in (40) and (41)] as the plotted lines tend to pivot about a rather stationary intercept at $\Delta=0$ (i.e. a value of about 0.9 or so). 'Jhus, it is easy to visualize that the slope in equation (40) (i.e. $0 \cdot 27$ ) would become smaller under the influence of prior cold work, thereby approaching a value of $0 \cdot 19-0 \cdot 22$ as per equation (39); the slope of equation (41) (i.e. 0.09) is already less than that given by (39). Because of the largo influence of $m$ in this analysis, it is difficult to explain this apparent contradiction.

Several observations are, however, offered in regard to the general findings expressed by (39). In that study, ${ }^{23}$ the condition of the starting metal (25 per cent cold work) would introduce a dependency of $\Phi$ on $m$ that is much less than that found with metals initially annealed. ${ }^{16}$ Since the influence of $\sigma_{0}$ on redundant work has been shown to be relatively small, therefore, one should not expect as great a variation in expressions for redundant work with prior-worked metals as compared to annealed metals. Additionally, the rango of $\Delta$ values up to 6 is somewhat restricted; in fact. the present authors noted greater scatter in their $\phi-\Delta$ plots ${ }^{16}$ at small values of $\Delta$ compared to larger values $(\Delta \approx 20)$ which arise by making small reductions with large die angles. According to Fig. 6.8 of his text, Row ${ }^{14}$ indicates that the smallest reduction used by Johnson ${ }^{23}$ was 19 per cent. In summary, therofore, one might conclude that experimenting with prior-worked metals over a small range of $\Delta$ could provide a number of test results that might best be described by a single line regardless of the metal employed. This may be an explanation to assist in interproting the possible reasons that lead to an equation such as (39); it does not, of course, explain why the discrepancy between the 0.09 value of equation $(41)$ and the $0 \cdot 19-0 \cdot 22$ value of $(39)$ exists.

\section{CONCLUSIONS}

(1) Work hardening effects in drawing theories can be handled either by the use of a proper mean yield stress or by incorporating strain hardening parameters in the basic force balance differential equation. For practical values of drawing parameters, these two approaches provide predictions of drawing stress that are very close; the use of a mean yield stress always establishes lesser magnitudes.

(2) The first conclusion would seem to be appropriate for other metalworking processes whose stress analyses are based upon a governing differential equation that is similar to that used in rod drawing (e.g. rolling and extrusion).

(3) Considering the validity of the "force balance" analysis in plasticity work, the small differences that arise, using the two approaches mentioned in the first conclusion, do not sensibly justify the extra mathematical complications associated with the more "exact" approach. Thus, the use of a proper mean yield stress seems quite adequate. 
(4) The redundant work factor determined by relating measured and predicted drawing stresses is not equivalent to the redundant work factor determined by the method of superposition of stress-strain curves.

(5) Jifferences between these two factors become more pronounced with metals possessing large strain hardening rates if the geometrical considerations (per cent reduction and die angle) are fixed.

(6) For a particular metal, differences between these two factors become more pronounced if small reductions are taken through large angle dies (i.e. large geometrical factor, $\Delta$ ).

Acknowledgements - Since this work was an extended effort of a previous study that was finencially assisted by the Institute of Science and Technology at the University of Michigan. the authors express their deep appreciation to that organization. Additionally, the correspondence of Dr. G. W. Rowe of the University of Birmingham, England proved enlightening and helpful. His provision of certain unpublished experimental results is accorded our sincere thanks.

\section{REFERENCES}

1. G. 1). S. MacLella , J. Iron Steel Inst. 158, 347 (1948).

2. J. G. Wistreich, Metall. Rev. 3, 97 (1958).

3. R. T. Shreld, J. Mech. Phys. Solids, 3, 246 (1955).

4. R. Hill and S. J. Tupper, J. Iron Steel Inst. 159, 353 (1848).

5. P. W. WhitToN, J. Inst. Metals 86, 417 (1957-58).

i. A. P. Green, Proc. Instn mech. Engrs 174, 847 (1960).

7. G. SACHs, $Z$. angew. Math. Mech. 7, 235 (1927).

8. F. Körber and A. Eichinger, Mitt. K.-Wilhelm-Inst. Eisenforsch, Dï̈seld. 22, 57 (1940).

9. E. A. Davis and S. J. Dokos, J. appl. Mech. 11, A193 (1944).

10. H. G. Baron and F. C. Thompson, J. Inst. Metals 78, 415 (1950-51).

11. G. Sachs and K. R. Van Horn, Practical Metallurgy, p. 391. Am. Soc. Metals (1940).

12. E. Voce, J. Inst. Metals 74, 537 (1948).

13. E. Voce, Metallurgia 51, 219 (1955).

14. G. W. Rowe, An Introduction to the Principles of Hetalworking. Edward Arnold, St. Martin's Press, New York (1965).

15. B. B. HUNDy and A. R. E. Singer, J. Inst. Metals 83, 401 (1954-55).

16. R. M. Caddeli and A. G. A'rkiss, Am. Soc. mech. Engrs to be published (1968).

17. W. Linices and G. SACHs, Mitt. Material, Sonderheft, 16, 38 (1931).

18. J. G. Wistreich, Proc. Instn mech. Engrs 169, 654 (1955).

19. E. Stebel, Stahl Eisen 66/67, 171 (1947).

20. G. D. S. MacLeLla , J. Inst. Metals 81, 1 (1952-53).

21. C. T. YA.G, Trans. Am. Soc. mech. Engrs B, J. Engng Ind. 83, 523 (1961).

22. P. R. Lancaster and G. W. Rowe, Proc. Inst. mech. Engrs 178, Part I, 69 (1963-64).

23. R. W. Johnsox, M.Sc. Dissertation, Birmingham University, Birmingham, England (1963).

24. G. W. Rowe, private communications (Fobruary and March 1966).

25. J. Datsko, Material Properties and Manufacturing Processes, p. 21. John Wiley, Now York (1966). 